\title{
Lock-in thermography with mechanical loss angle heating at ultrasonic frequencies
}

\author{
by J. Rantala ${ }^{2}$, D. Wu ${ }^{1}$, A. Salerno ${ }^{1}$, G. Busse ${ }^{1}$ \\ 'Institut für Kunststoffprüfung und Kunststoffkunde, Universität Stuttgart, Pfaffenwaldring 32, D-70569 \\ Stuttgart, Germany \\ ${ }^{2}$ The Academy of Finland, P.O. Box 57, 00551 Helsinki, Finland
}

\begin{abstract}
In this paper the mechanical loss angle heating as an internal thermal wave source is described. Acoustic energy at high frequency (ultrasound) can be coupled into the sample. The high hysteresis loss in the defect region results in increased heating of the defect. When the intensity of the ultrasound is modulated at a low frequency the defect itself emits a thermal wave at this low frequency. The range of this thermal wave is large enough for detection at the surface with lock-in thermography tuned to the intensity modulation. This technique combining ultrasonic waves and lock-in thermography provides a fast imaging of imaginary part of Young's modulus in materials. Results obtained with this selective heating on various real components are presented.
\end{abstract}

\section{Introduction}

Temperature change in materials can be induced with mechanical vibration where the thermo-elastic effect and hysteresis effect are involved [1]. As known the SPATE measurement ("stress pattern analysis by thermal emission") based on thermo-elastic effect provides information about the stress distribution in a component. The thermo-elastic effect is the dominating mechanism in metals. In polymers, however, the hysteresis loss dominates already at low amplitudes, as is obvious from their high acoustic or mechanical damping. Defects are usually areas of stress concentration which causes a local increase of imaginary part of Emodul. After one loading cycle one obtains a hysteresis loop in the stress/strain diagram. The enclosed area corresponds to the dissipated energy which is converted into heat. The heat generation in material due to hysteresis effect can be monitored with a thermography camera [2]. As the rate of heat generation is propotional to the vibration frequency, it is more efficient to use high frequency mechanical vibration e.g. ultrasound. The advantage of ultrasonic heating is that a large thermal signal can be obtained at a low stress level [3]. Since the temperature increase in the defect area is much higher than in its environment, the defect can be well detected using thermography like "dark field microscopy". Therefore, the use of the ultrasonic heating provides a method where a defect can be detected selectively [4]. By applying a low frequency amplitude modulation to the high frequency ultrasonic vibration one obtains a modulated heat generation which is detected with lock-in thermography where one obtains phase and amplitude images. As the phase contrast mechanism is the phase lag of the temperature modulation referred to the amplitude modulation, effects of inhomogeneous heating due to acoustic damping along the wave propagation and the variation of the surface emissivity are suppressed in the phase image. Information on defect depth can be derived from signal phase.

\section{Results}

The coupling of ultrasound into the samples was performed using mechanical contact. The electrical power of the ultrasound generator is $150 \mathrm{~W}$. Ultrasonic transducers oscillating at 40 $\mathrm{kHz}$ were intensity modulated at $0.03 \mathrm{~Hz}$. The measurement setup is shown in Fig. 1. For the 
inspection of layered materials various kinds of subsurface defects are investigated with lock-in vibro-thermography at ultrasound frequency.

\subsection{Wood}

Delaminations can occur in the manufacturing process of wood-based panels with coating materials such as veneer. These defects have to be detected in an early stage. A sample shown in Fig. 2 was investigated. Between the $0.5 \mathrm{~mm}$ veneer layer and substrate there are several holes and two embedded sheets: a piece of teflon film in the middle and an aluminium film at the right bottom corner. All these defects can be clearly detected in the phase and amplitude image.

\subsection{Polymers}

The quality of polymer materials may suffer from boundary effects. Therefore one is interested to detect boundaries or their changes. One example is welding of polymers. Areas of disbond are inherent sources of weakness which result in failure under load. The results of a welded GFRP sample are displayed in Fig. 3. In the middle of the sample there is an air gap between two bonded parts. Defects were shown up in the phase and amplitude image when ultrasonic waves are coupled into the sample. The contrast of phase and amplitude image are much better than in a thermographic image.

CFRP laminates have excellent mechanical properties. Since the anisotropy is easily controllable with the orientation of plies, one can optimise the stiffness with respect to certain kinds of load. However, this stiffness depends on the quality of boundaries between the unidirectional plies. One kind of defect is impact damage resulting in delaminations and cracks that propagate along plies. Such impact damages are usually invisible on the impact side.

Fig 4 shows the results of lock-in vibro-thermography by using an ultrasonic transducer on a CFRP sample with 7 impact damages. In this case the ultrasonic source is located on the left of the sample. The phase image shows 7 impact damages clearly. Due to attenuation of the ultrasound intensity along the propagation way, the amplitude image shows how the appearance of defects depends on the local ultrasound intensity.

\subsection{Ceramics}

Intact boundaries are also essential for coated materials where the outer layers are supposed to have some special functions, e.g. improved resistance to wear, corrosion, or heat. In these cases one is interested to monitor local variations of thickness or to detect areas of disbond where the loss of adhesion may later on result in failure. A metal sample with ceramic coating on a metal substrate was inspected by using lock-in vibro-thermography. A delamination area on the upper edge of the sample (Fig. 5) is detected. In the phase image the beginning of the delamination can be seen clearly.

For health-monitoring of safety relevant components one needs to detect cracks in their early stages of generation. When cracks are vertical to the surface they are usually difficult to be detected, especially when its thermal contact is very good. In these cases ultrasonic heating provides a powerful tool for crack imaging. Vertical cracks are heated up due to friction between two parts of cracks and result in appearing in the thermographic image. Fig. 6 shows an example where a vertical crack in a ceramic component can be revealed in a dark field fashion. 


\subsection{Metal}

The quality of metal can be reduced by corrosion. This is a serious problem e.g. for aging aircraft. The mechanical loss angle effect increases in corroded areas as well. Fig. 7 shows lock-in vibro-thermography results obtained on an airplane component (aluminium) with hidden corrosion (due to humidity and high mechanical stresses) on its rear side. The corrosion depth is about $15 \%$ of the whole thickness $(4 \mathrm{~mm})$. Phase and amplitude image reveal just the corrosion defect without any influence of wall thickness variations (which may be seen in phase and amplitude image of lock-in thermography operating with external heat sources).

\section{Summary}

The feasibilty of lock-in vibro-thermography at ultrasonic frequency was demonstrated with various kinds of defects in different materials. The examples show that disbonding, delamination, crack, and corrosion in materials can be detected in a dark field fashion.

Lock-in thermography combines ultrasonic waves, fast imaging facilities of thermography and the maginary part of Young's modulus in materials to provide a technique which will find many applications for in-service inspection of industrial components.

\section{Acknowledgement}

The authors are grateful to German BMBF for support of research on lock-in thermography (Project Nr. 03N8006B0). One author (J. R.) is indebted to the Alexander von HumboldtFoundation for granting a fellowship and one author (A. S.) to Foundation BLANCEFLOR Boncompagni-Ludovisi, née Bildt for granting a fellowship. All authors appreciate the efficient cooperation with AGEMA.

\section{REFERENCES}

[1]BUSSE (G.), BAUER (M.), RIPPEL (W.), and WU (D.). - Lockin vibrothermal inspection of polymer composites. QIRT'92, Editions Européennes Thermique et Industrie, Paris, 1992 p. 154

[2] HENNEKE (E. G.), REIFSNIDER (K. L.), and STINCHCOMB (W. W.). - Thermography $\bigcirc$ An NDI method for damage detection. Journal of Metals, 31, 1979, p. 11

[3] RANTALA (J.), WU (D.), and BUSSE (G.). - Amplitude modulated lock-in vibrothermography for NDE of polymers and composites. Research in Nondestructive Evaluation 7, 1996, p. 215

[4] RANTALA (J.), WU (D.), and BUSSE (G.). - NDT of polymer materials using lock-in thermography with water-coupled ultrasonic excitation. NDT\&E International (in print). 


\section{http://dx.doi.org/10.21611/qirt.1996.064}

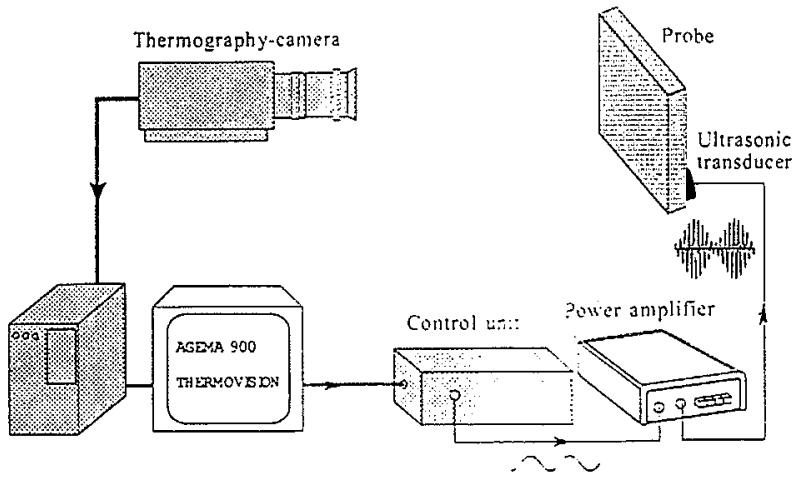

Fig. 1. Schematical diagram of ultrasonic lock-in vibro-thermography

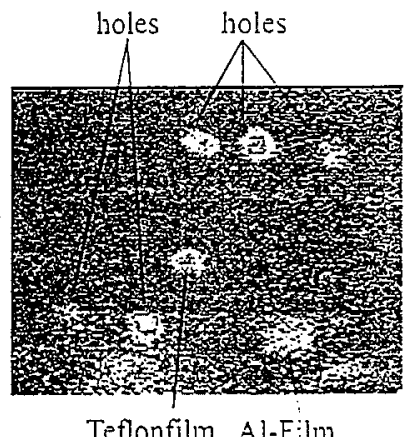

A -F: $\ln$

Amplitude $(0.03 \mathrm{~Hz})$

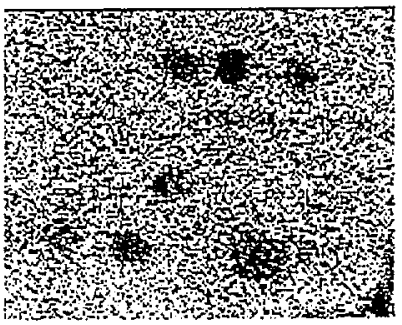

Phase $(0,03 \mathrm{~Hz})$

Fig. 2. Veneered wood with various defests under veneer layer

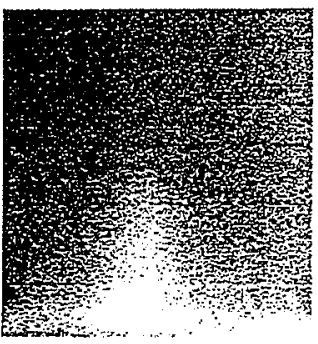

Thermography

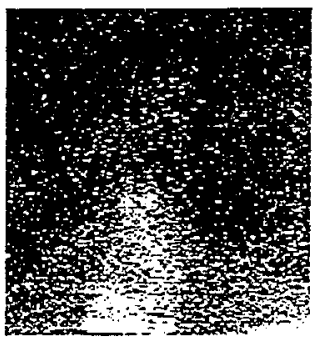

Amplitude $(0.03 \mathrm{~Hz})$

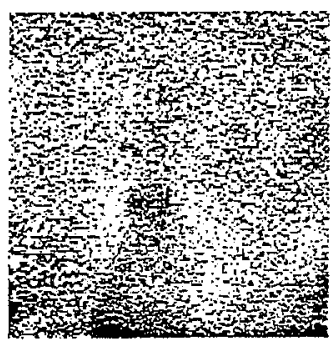

Phase $(0.03 \mathrm{~Hz})$

Fig.3. Two welded GFRP-plates with bc-idirg defect (air gap with wedge form in the middle of images) 


\section{http://dx.doi.org/10.21611/qirt.1996.064}
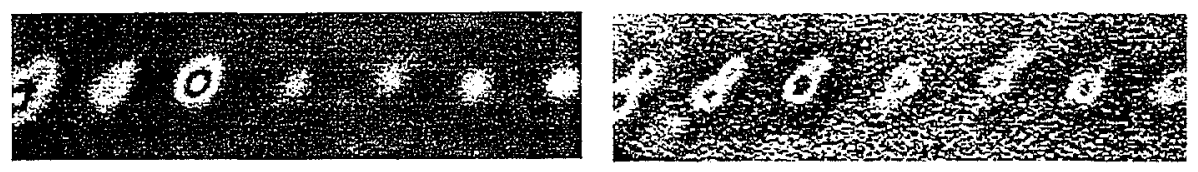

Fig. 4. Amplitude (left) and phase (right) image of a CFRP sample with 7 impact damages
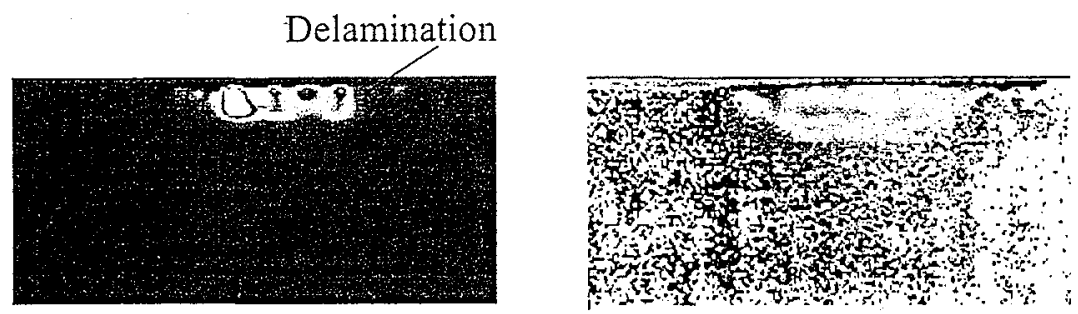

Fig. 5. Amplitude (left) and phase (right) of a metal sample with ceramic coating on it
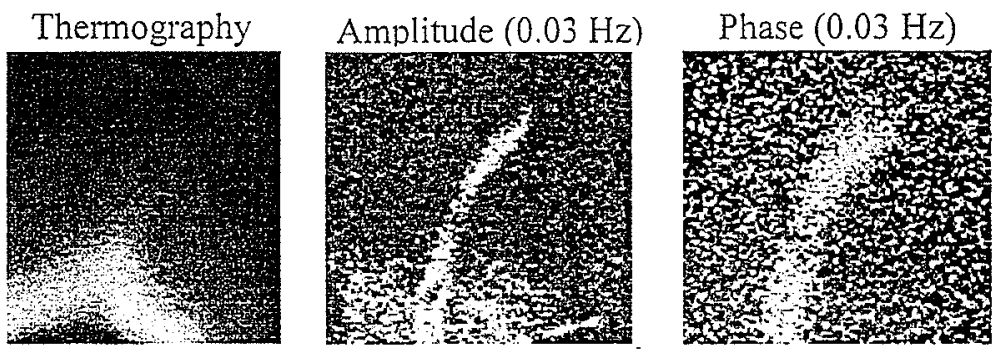

Fig. 6. Detection of a vertical crack in a ceramic component using ultrasound excitation

Amplitude $(0.12 \mathrm{~Hz})$

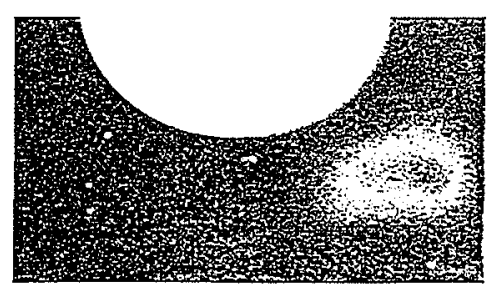

Phase $(0.12 \mathrm{~Hz})$

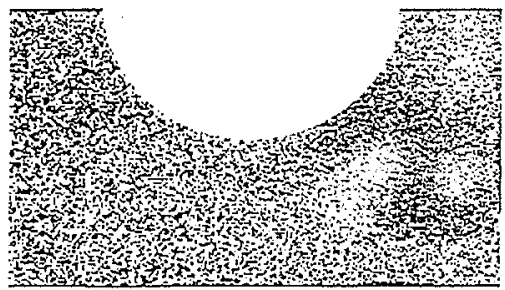

Fig. 7. Selective heating of corroded area in an aluminium airplane component 